\title{
Is it Fabry disease?
}

\author{
Raphael Schiffmann, MD, MHSc', Maria Fuller, PhD², Lorne A. Clarke, MD³ and \\ Johannes M.F.G. Aerts, $\mathrm{PhD}^{4}$
}

Fabry disease is caused by mutations in the GLA gene that lower a-galactosidase A activity to less than $25-30 \%$ of the mean normal level. Several GLA variants have been identified that are associated with relatively elevated residual $\alpha$-galactosidase A. The challenge is to determine which GLA variants can cause clinical manifestations related to Fabry disease. Here, we review the various types of GLA variants and recommend that pathogenicity be considered only when associated with elevated globotriaosylceramide in disease-relevant organs and tissues as analyzed by mass spectrometry. This criterion is necessary to ensure that very costly and specific therapy is provided only when appropriate.

Genet Med advance online publication 19 May 2016

Key Words: benign variant; GLA gene; lysosomal storage disease; risk factor
Advances in the past decade have led to the elucidation of the genetic and metabolic basis of an extensive array of rare and often devastating diseases, which has been crucial for the development of therapeutics for those diseases. Genotype-phenotype correlative studies in these rare disorders and the identification of common allelic variants have provided interesting insights into the potential role that rare disease gene variants may play in the pathogenesis of more common disease symptoms within the general population.

Fabry disease is caused by mutations of the X-linked GLA gene that produce a deficiency of the lysosomal enzyme $\alpha$-galactosidase A. ${ }^{1}$ Deficiency of $\alpha$-galactosidase A results in the inability of cells to catabolize glycosphingolipids with terminal $\alpha$-D-galactosyl residues. ${ }^{2}$ These glycosphingolipids, particularly globotriaosylceramide $\left(\mathrm{Gb}_{3}\right)$, progressively accumulate in virtually all organs, resulting in a progressive multisystem disease. The cardinal features of the disease are acroparesthesias, progressive proteinuric renal insufficiency, cardiac disease consisting of rhythm and conduction disturbances and progressive hypertrophic cardiomyopathy, as well as cerebrovascular stroke. Patients with the classic form of Fabry disease have very low, if any, residual enzyme activity. ${ }^{3}$ Over the years, attenuated forms of the disease have been increasingly recognized. Importantly, patients with attenuated disease have clinically significant disease, with cardiac involvement being the most common manifestation. In addition, patients with attenuated disease tend to have higher enzyme activity and a more protracted disease natural history than patients with classic disease. ${ }^{4}$

The incidence of Fabry disease is approximately 1 in 117,000 live births for males, ${ }^{5}$ although recent newborn screening surveys suggest that the incidence may be much higher (up to $1: 3,100) .{ }^{6-11}$ In Taiwan, the incidence of the relatively mild IVS4+919G $>$ A mutation is 1:875 male live births and 1:399 female live births. ${ }^{11}$

Screening of various at-risk patient populations, i.e., patients with cardiomyopathy, ${ }^{12,13}$ stroke, ${ }^{14}$ or renal failure, ${ }^{15,16}$ has identified a low, but not negligible, prevalence of Fabry disease in the general symptomatic population. Some of these variants have normal or almost normal enzyme activity and, in other cases, mildly to moderately reduced $\alpha$-galactosidase A activity. These observations pose two fundamental questions: (i) do patients identified in this manner (many of whom have common symptoms, i.e., stroke, cardiomyopathy, proteinuria, or renal failure) have Fabry disease? and (ii) should $\alpha$-galactosidase enzyme replacement or other Fabry-specific therapies be considered in their management?

Because of the recognition of frequent GLA variants, it has become important to establish criteria by which a patient with or without symptoms and a specific variant would be considered to have or to be at risk for Fabry-related complications. Here, we discuss problems related to the interpretation of GLA variants and propose criteria for designating pathogenicity in the clinical context. Determination of the true significance of a GLA variant is critical to apply specific therapy only when indicated.

\section{DIAGNOSIS OF FABRY DISEASE}

The diagnosis of Fabry disease requires the demonstration of enzyme deficiency and a resultant increase in cell/organ globotriaosylceramide, typically in lysosomes. ${ }^{17}$ It is the accumulation of substrate that initiates pathological cascades, which ultimately are responsible for patient symptoms rather than the enzyme deficiency per se. ${ }^{2}$ Therefore, ensuring that the increase

${ }^{1}$ Institute of Metabolic Disease, Baylor Research Institute, Dallas, Texas, USA; ${ }^{2}$ Genetics and Molecular Pathology, SA Pathology at Women's and Children's Hospital, North Adelaide, Australia; ${ }^{3}$ Department of Medical Genetics, Child and Family Research Institute, University of British Columbia, Vancouver, British Columbia, Canada; ${ }^{4}$ Department of Medical Biochemistry, Leiden University, Leiden, The Netherlands. Correspondence: Raphael Schiffmann (Raphael.schiffmann@BSWhealth.org)

Submitted 18 January 2016; accepted 16 March 2016; advance online publication 19 May 2016. doi:10.1038/gim.2016.55 
of this glycosphingolipid is present in a relevant organ system is central to dealing with whether the patient has Fabry disease or is at risk for Fabry disease-related complications.

\section{WHAT IS THE THRESHOLD FOR CLINICALLY SIGNIFICANT $\alpha$-GALACTOSIDASE A DEFICIENCY?}

Patients with the classic form of Fabry disease have no residual enzyme activity., ${ }^{4,18}$ However, the enzyme activity level above which $\alpha$-galactosidase A deficiency does not play a role in human disease is not known. It has been estimated that residual activity of $30-35 \%$ of mean normal $\alpha$-galactosidase $\mathrm{A}$ activity is the cutoff for diagnosing Fabry disease. ${ }^{11,13,19,20}$ Enzymatic activity is being routinely measured in isolated peripheral blood white cells or in dry blood spots. However, $\alpha$-galactosidase A activity in peripheral blood may not reflect the activity in affected organs such as the kidney, heart, and vascular system. The true threshold of pathogenicity for Fabry disease is not known. Although such a threshold has been claimed for other diseases, ${ }^{21}$ it may vary from organ to organ and from one patient to another. The frequent observation of exclusive cardiac involvement in Fabry disease suggests that the heart is the most susceptible organ to $\alpha$-galactosidase A deficiency. ${ }^{22,23}$ It is important to note that although clinically significant renal disease is often not detected in Fabry patients with exclusive cardiac disease, it is not known whether renal storage of glycolipids is present in these patients. Exclusive kidney disease has very rarely been described. Clinical observation suggests that patients with the classic form of Fabry disease are those who are at the highest risk for stroke. Therefore, a heart-kidney-cerebrovascular system susceptibility gradient is likely.

\section{INTERACTION OF A-GALACTOSIDASE DEFICIENCY WITH OTHER FACTORS}

The clinical heterogeneity noted in Fabry individuals carrying the same pathogenic mutation, even within the same family, is clear evidence that an individual's risk for complications of Fabry disease depends on interactions between $\alpha$-galactosidase A deficiency and other factors. The additional factors could include genetic, epigenetic, and environmental factors. In a threshold model of symptom emergence, the higher the residual enzyme activity, the greater the influence these other factors probably have on the likelihood of developing Fabry-related clinical complications. Little work has been done to identify genetic modifiers in Fabry disease. Genotypes of polymorphisms G-174C of interleukin-6, G894T of endothelial nitric oxide synthase, factor V G1691A mutation (factor V Leiden), and the A-13G and G79A of protein $\mathrm{Z}$ were all significantly associated with the presence of presumably ischemic cerebral lesions on brain magnetic resonance imaging (MRI). ${ }^{24}$ Using the mouse model for Fabry disease, it was shown that the absence of $\alpha$-galactosidase A activity combined with the factor $\mathrm{V}$ Leiden mutation significantly increases the number of vascular thrombi compared to either mouse model alone. ${ }^{25}$ The role of factor $\mathrm{V}$ Leiden mutation in increasing the likelihood of thromboembolic events was subsequently confirmed. ${ }^{26}$

\section{GLA VARIANTS AND CLINICAL EXPRESSION}

One can divide GLA variants into three categories of $\alpha$-galactosidase A residual activity (Figure 1). Nonsense and certain missense variants that are virtually always associated with Fabry disease have a level of enzyme activity of 0 to approximately $10 \%$ of normal in males. ${ }^{27,28}$ In this case, nonGLA modifiers (genetic, epigenetic, or other) have relatively little effect on the likelihood of having Fabry-related clinical complications. Such complications are less common in those with missense and certain splice GLA variants, with residual enzyme activity in the range of $15-30 \%$ of normal in males. ${ }^{3}$ The clinical expression of this second group likely depends, to a large extent, on putative genetic and epigenetic modifiers. The third group consists of GLA variants that are not associated with substantially reduced enzyme activity, i.e., activity above $35-40 \%$ of mean normal controls in males. In this group of GLA variants, clinical complications similar to those that occur in Fabry disease are more likely to be unrelated to the below average activity level of $\alpha$-galactosidase $A$. The most controversial group of variants seems to be the nonpathogenic variants. A few examples illustrate the pitfalls associated with identification of such variants in patients. A 7-year-old boy with neuropathic pain was found to have the S126G variant; therefore, he was diagnosed as having Fabry disease. This variant was identified in a screening of females only and was considered pathogenic. ${ }^{29}$ However, enzyme activity in this hemizygous male patient was found to be normal by two national laboratories (A. Basinger, unpublished data) and is considered benign or tolerated by prediction tools such as Sorting Intolerant From Tolerant and PolyPhen. D313Y was initially described as a disease-causing mutation ${ }^{27}$ and has been associated with stroke $e^{30}$ and white matter damage. ${ }^{31}$ However, previous publications found lower than normal plasma activity due to reduced activity in neutral $\mathrm{pH}$, whereas activity in white blood cells was normal or minimally reduced. ${ }^{32,33}$ Normal lyso- $\mathrm{Gb}_{3}$ in patients was considered proof that this variant is not pathogenic. ${ }^{34}$ The $\mathrm{R} 118 \mathrm{C}$ is considered a pathogenic mutation in a number of publications. ${ }^{14,35}$ Male patients with the R118C variant have mean $\alpha$-galactosidase levels of $32 \%$ of normal. ${ }^{19}$ No zebra bodies (lysosomal inclusions) were found in a cardiac biopsy specimen of a male patient with this variant and no tissue $\mathrm{Gb}_{3}$

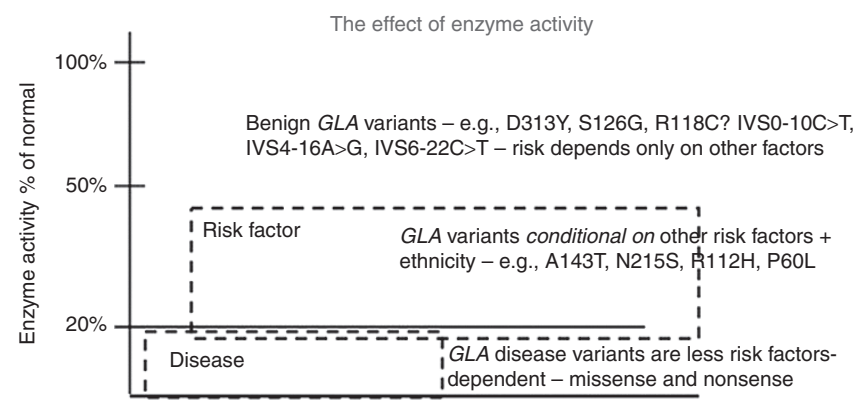

Figure 1 The relationship between the various types of GLA gene variants and the residual $\alpha$-galactosidase $A$ activity. 
levels were measured. ${ }^{19}$ As can be gleaned from Figure 4 in the work by Ferreira et al. ${ }^{19}$, it is possible that the lowered residual enzyme activity may be sufficient to contribute to cardiovascular or renal disease in some patients with other risk factors. This concept has sometimes been called synergistic heterozygosity. ${ }^{36,37} \mathrm{~A}$ number of intronic variants have been described in male and female patients with stroke, cerebral white matter abnormality, and small-fiber neuropathy. ${ }^{38}$ In addition, $-10 \mathrm{C}>\mathrm{T}$ is associated with reduced GLA expression and, consequently, mildly reduced $\alpha$-galactosidase A activity (reduced by approximately $15-20 \%$ but not in disease range). ${ }^{39}$ Therefore, $-10 \mathrm{C}>\mathrm{T}$ is likely to be a disease modifier when associated with a pathogenic variant. Of note, symptomatic $-10 \mathrm{C}>\mathrm{T}$ variant patients have been administered ERT, resulting in apparent reduction in neuropathic pain and increased physical activity ${ }^{38}$.

The association of clinical manifestations in the presence of the exonic variants such as D313Y or intronic variants cannot be explained solely based on deficiency of $\alpha$-galactosidase A. Studies that do show such associations ${ }^{31}$ have not demonstrated increased $\mathrm{Gb}_{3}$ in tissues; therefore, the clinical findings are more likely due to other genetic or environmental factors and may or may not be linked to the variant allele.

\section{HOW TO DETERMINE THE PATHOGENICITY OF A GLA VARIANT}

It is clear from this discussion that the level of enzyme activity alone is not always sufficient to determine whether a particular GLA variant is pathogenic (Figure 2). Unfortunately, there are no databases that capture accurate and comprehensive Fabry-related clinical findings in association with GLA variants. Nevertheless, when a GLA variant is identified, databases such as ClinVar and the Exome Variant Server (Exome Variant Server, NHLBI GO Exome Sequencing Project, Seattle, WA; http://evs.gs.washington.edu/EVS/) should be interrogated to determine the allele frequency and potential phenotypes. If these databases are proven to be not helpful, then other approaches are suggested. The most commonly invoked is the demonstration of substrate accumulation or altered sphingolipid levels, i.e., $\mathrm{Gb}_{3}$ or lyso-Gb3 in plasma or urine. ${ }^{34,40,41}$ However, $\mathrm{Gb}_{3}$ and lyso- $\mathrm{Gb}_{3}$ have limitations because both may be normal in attenuated forms of the disease or in females. ${ }^{40,42}$ Conversely, lyso- $\mathrm{Gb}_{3}$ was recently found to be increased in plasma of untreated patients with Gaucher disease type 1 to the range seen in patients with Fabry disease ${ }^{43,44}$ and in heart disease patients who do not have any GLA variants and have normal enzyme activity. ${ }^{45}$ Alternatively, identification of typical lysosomal inclusions in tissue biopsy specimens has been suggested as the gold standard of Fabry disease diagnosis. ${ }^{46,47}$ In our view, although its presence suggests Fabry disease, it is not the ideal criterion. First, lysosomal inclusions (sometime referred to as zebra bodies) are not specific to Fabry disease. They may occur in other lysosomal storage diseases such as GM2 gangliosidosis and Niemann-Pick disease and have also been demonstrated in silicon nephropathy. ${ }^{48,49}$ Conversely, $\mathrm{Gb}_{3}$ level may be increased in the absence of lysosomal inclusions..$^{50,51}$

The most specific way to assess whether $\alpha$-galactosidase A deficiency is clinically significant in a particular individual is to demonstrate the presence of an increased tissue level of this enzyme's direct substrate $\left(\mathrm{Gb}_{3}\right)$ Ideally, a disease-relevant organ such as the kidney or the heart should be assessed using

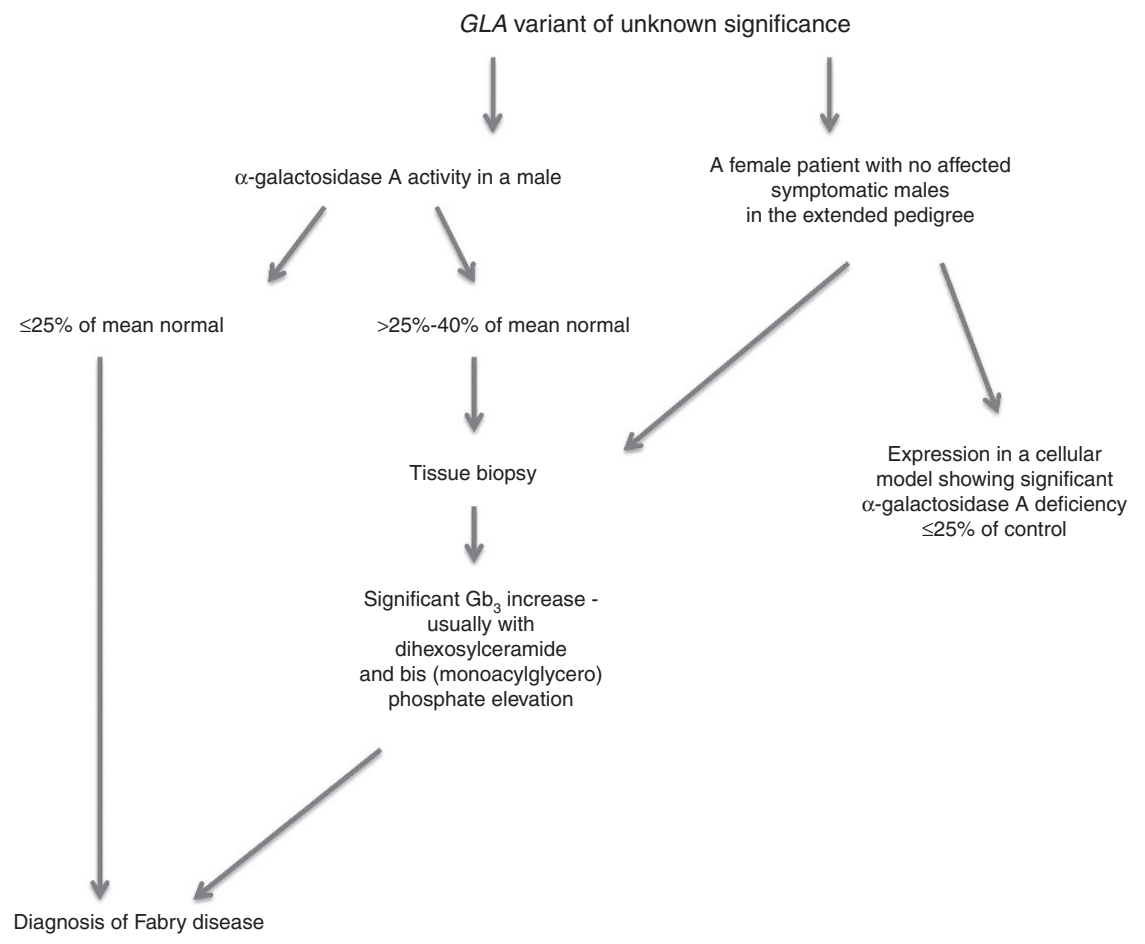

Figure 2 An algorithm for evaluating a GLA variant of unknown significance. 
mass spectrometry, but skin may be adequate in some cases (Figure 2). ${ }^{52} \mathrm{~Gb}_{3}$ elevation can also be demonstrated by immunohistochemistry in a tissue biopsy specimen using anti- $\mathrm{Gb}_{3}$ antibody. ${ }^{50,51}$

\section{CONCLUSIONS}

The nonspecific nature of the complications of Fabry disease and the relatively high population frequency of Fabry disease are risks for the wrong attribution of pathogenicity to certain GLA variants when they are identified in such patients. This can lead to unnecessary initiation of expensive and invasive therapies such as ERT instead of appropriately addressing the true causes of cerebrovascular, cardiac, renal, and neurological manifestations. Therefore, before concluding that a variant with relatively high $\alpha$-galactosidase A activity is pathogenic, one must demonstrate evidence of altered sphingolipid homeostasis. Demonstration of elevated $\mathrm{Gb}_{3}$ using mass spectroscopy is critical. When the specificity of the $\mathrm{Gb}_{3}$ elevation is in doubt, the presence of a Fabry-specific lipid profile is likely to be helpful. ${ }^{53}$

\section{DISCLOSURE}

R.S. received research grants, honoraria, and travel expenses from Amicus Therapeutics, Protalix Biotherapeutics, and Shire. He also gives talks on behalf of Genzyme (A Sanofi Company). The other authors declare no conflict of interest.

\section{REFERENCES}

1. Brady RO, Gal AE, Bradley RM, Martensson E, Warshaw AL, Laster L. Enzymatic defect in Fabry's disease. Ceramidetrihexosidase deficiency. N Engl J Med 1967;276:1163-1167.

2. Sweeley CC, Klionsky B. Fabry's disease: classification as a sphingolipidosis and partial characterization of a novel glycolipid. J Biol Chem 1963;238:3148-3150.

3. Eng CM, Ashley GA, Burgert TS, Enriquez AL, D'Souza M, Desnick RJ. Fabry disease: thirty-five mutations in the alpha-galactosidase $A$ gene in patients with classic and variant phenotypes. Mol Med 1997;3:174-182.

4. Branton MH, Schiffmann R, Sabnis SG, et al. Natural history of Fabry renal disease: influence of alpha-galactosidase A activity and genetic mutations on clinical course. Medicine (Baltimore) 2002;81:122-138.

5. Meikle PJ, Hopwood JJ, Clague AE, Carey WF. Prevalence of lysosomal storage disorders. JAMA 1999;281:249-254.

6. Spada M, Pagliardini S, Yasuda M, et al. High incidence of later-onset fabry disease revealed by newborn screening. Am J Hum Genet 2006;79:31-40.

7. Liao HC, Chiang CC, Niu DM, et al. Detecting multiple lysosomal storage diseases by tandem mass spectrometry-a national newborn screening program in Taiwan. Clin Chim Acta 2014;431:80-86.

8. Lin HY, Chong KW, Hsu JH, et al. High incidence of the cardiac variant of Fabry disease revealed by newborn screening in the Taiwan Chinese population. Circ Cardiovasc Genet 2009;2:450-456.

9. Inoue T, Hattori K, Ihara K, Ishii A, Nakamura K, Hirose S. Newborn screening for Fabry disease in Japan: prevalence and genotypes of Fabry disease in a pilot study. J Hum Genet 2013;58:548-552.

10. van der Tol L, Cassiman D, Houge G, et al. Uncertain diagnosis of fabry disease in patients with neuropathic pain, angiokeratoma or cornea verticillata: consensus on the approach to diagnosis and follow-up. JIMD Rep 2014;17:83-90.

11. Chien YH, Lee NC, Chiang SC, Desnick RJ, Hwu WL. Fabry disease: incidence of the common later-onset $\alpha$-galactosidase A IVS4+919G $\rightarrow$ A mutation in Taiwanese newborns-superiority of DNA-based to enzyme-based newborn screening for common mutations. Mol Med 2012;18:780-784.

12. Sachdev B, Takenaka T, Teraguchi $\mathrm{H}$, et al. Prevalence of Anderson-Fabry disease in male patients with late onset hypertrophic cardiomyopathy. Circulation 2002;105:1407-1411.

13. Monserrat L, Gimeno-Blanes JR, Marín F, et al. Prevalence of fabry disease in a cohort of 508 unrelated patients with hypertrophic cardiomyopathy. J Am Coll Cardio/ 2007;50:2399-2403.
14. Rolfs A, Fazekas F, Grittner U, et al. Acute cerebrovascular disease in the young: the stroke in young fabry patients study. Stroke 2013;44:340-349.

15. Wallin EF, Clatworthy MR, Pritchard NR. Fabry disease: results of the first UK hemodialysis screening study. Clin Nephrol 2011;75:506-510.

16. Doi K, Noiri E, Ishizu T, et al. High-throughput screening identified diseasecausing mutants and functional variants of $\alpha$-galactosidase A gene in Japanese male hemodialysis patients. J Hum Genet 2012;57:575-579.

17. Desnick RJ, Allen KY, Desnick SJ, Raman MK, Bernlohr RW, Krivit W. Fabry's disease: enzymatic diagnosis of hemizygotes and heterozygotes. Alphagalactosidase activities in plasma, serum, urine, and leukocytes. J Lab Clin Med 1973;81:157-171.

18. Sakuraba $H$, Oshima A, Fukuhara $Y$, et al. Identification of point mutations in the alpha-galactosidase $A$ gene in classical and atypical hemizygotes with Fabry disease. Am J Hum Genet 1990;47:784-789.

19. Ferreira S, Ortiz A, Germain DP, et al. The alpha-galactosidase A p.Arg118Cys variant does not cause a Fabry disease phenotype: data from individual patients and family studies. Mol Genet Metab 2015;114:248-258.

20. Andrade J, Waters PJ, Singh RS, et al. Screening for Fabry disease in patients with chronic kidney disease: limitations of plasma alpha-galactosidase assay as a screening test. Clin J Am Soc Nephro/ 2008;3:139-145.

21. Leinekugel P, Michel S, Conzelmann E, Sandhoff K. Quantitative correlation between the residual activity of beta-hexosaminidase $A$ and arylsulfatase A and the severity of the resulting lysosomal storage disease. Hum Genet 1992:88:513-523.

22. Chimenti C, Ricci R, Pieroni M, Natale L, Russo MA, Frustaci A. Cardiac variant of Fabry's disease mimicking hypertrophic cardiomyopathy. Cardiologia 1999;44:469-473.

23. Havndrup O, Christiansen M, Stoevring B, et al. Fabry disease mimicking hypertrophic cardiomyopathy: genetic screening needed for establishing the diagnosis in women. Eur J Heart Fail 2010;12:535-540.

24. Altarescu G, Moore DF, Schiffmann R. Effect of genetic modifiers on cerebral lesions in Fabry disease. Neurology 2005;64:2148-2150.

25. Shen Y, Bodary PF, Vargas FB, et al. Alpha-galactosidase A deficiency leads to increased tissue fibrin deposition and thrombosis in mice homozygous for the factor V Leiden mutation. Stroke 2006;37:1106-1108.

26. Lenders $M$, Karabul N, Duning T, et al. Thromboembolic events in Fabry disease and the impact of factor V Leiden. Neurology 2015;84:1009-1016.

27. Eng CM, Resnick-Silverman LA, Niehaus DJ, Astrin KH, Desnick RJ. Nature and frequency of mutations in the alpha-galactosidase A gene that cause Fabry disease. Am J Hum Genet 1993;53:1186-1197.

28. Eng CM, Desnick RJ. Molecular basis of Fabry disease: mutations and polymorphisms in the human alpha-galactosidase A gene. Hum Mutat 1994;3:103-111.

29. Pasqualim G, Simon L, Sperb-Ludwig F, et al. Fabry disease: a new approach for the screening of females in high-risk groups. Clin Biochem 2014;47: 657-662.

30. Baptista MV, Ferreira S, Pinho-E-Melo T, et al.; PORTuguese Young STROKE Investigators. Mutations of the GLA gene in young patients with stroke: the PORTYSTROKE study-screening genetic conditions in Portuguese young stroke patients. Stroke 2010;41:431-436.

31. Lenders M, Duning T, Schelleckes $M$, et al. Multifocal white matter lesions associated with the D313Y mutation of the $\alpha$-galactosidase A gene. PLoS One 2013;8:e55565.

32. Yasuda M, Shabbeer J, Benson SD, Maire I, Burnett RM, Desnick RJ. Fabry disease: characterization of alpha-galactosidase A double mutations and the D313Y plasma enzyme pseudodeficiency allele. Hum Mutat 2003;22:486-492.

33. Froissart R, Guffon N, Vanier MT, Desnick RJ, Maire I. Fabry disease: D313Y is an alpha-galactosidase A sequence variant that causes pseudodeficient activity in plasma. Mo/ Genet Metab 2003;80:307-314.

34. Niemann M, Rolfs A, Giese A, et al. Lyso-Gb3 indicates that the alphagalactosidase a mutation $\mathrm{D} 313 \mathrm{Y}$ is not clinically relevant for Fabry disease. JIMD Rep 2013;7:99-102.

35. Caetano F, Botelho A, Mota P, et al. Fabry disease presenting as apical left ventricular hypertrophy in a patient carrying the missense mutation R118C. Rev Port Cardiol 2014;33:183 e181-185.

36. Vockley J, Rinaldo P, Bennett MJ, Matern D, Vladutiu GD. Synergistic heterozygosity: disease resulting from multiple partial defects in one or more metabolic pathways. Mol Genet Metab 2000;71:10-18.

37. Phillips JA 3rd, Poling JS, Phillips CA, et al. Synergistic heterozygosity for TGFbeta1 SNPs and BMPR2 mutations modulates the age at diagnosis and penetrance of familial pulmonary arterial hypertension. Genet Med 2008; 10:359-365. 
38. Schelleckes M, Lenders M, Guske K, et al. Cryptogenic stroke and small fiber neuropathy of unknown etiology in patients with alpha-galactosidase A -10T genotype. Orphanet J Rare Dis 2014;9:178

39. Desnick RJ, Doheny DO, Chena B, et al. Fabry disease: The $\alpha$-galactosidase A (GLA) c.427GNA (A143T) mutation, effect of the $5^{\prime}$-10CNT polymorphism. Mol Genet Metab 2015; 114: S37.

40. Aerts JM, Groener JE, Kuiper S, et al. Elevated globotriaosylsphingosine is a hallmark of Fabry disease. Proc Natl Acad Sci USA 2008;105:2812-2817.

41. Niemann M, Rolfs $A$, Störk $S$, et al. Gene mutations versus clinically relevant phenotypes: lyso-Gb3 defines Fabry disease. Circ Cardiovasc Genet 2014;7: 8-16.

42. Smid BE, van der Tol L, Biegstraaten M, Linthorst GE, Hollak CE, Poorthuis BJ Plasma globotriaosylsphingosine in relation to phenotypes of Fabry disease. J Med Genet 2015;52:262-268.

43. Ferraz MJ, Marques AR, Appelman MD, et al. Lysosomal glycosphingolipid catabolism by acid ceramidase: formation of glycosphingoid bases during deficiency of glycosidases. FEBS Lett 2016;590:716-725.

44. Mirzaian M, Wisse $P$, Ferraz MJ, et al. Mass spectrometric quantification of glucosylsphingosine in plasma and urine of type 1 Gaucher patients using an isotope standard. Blood Cells Mol Dis 2015:54:307-314

45. Schiffmann R, Forni S, Swift C, et al. Risk of death in heart disease is associated with elevated urinary globotriaosylceramide. J Am Heart Assoc 2014;3:e000394.
46. Smid BE, van der Tol L, Cecchi F, et al. Uncertain diagnosis of Fabry disease: consensus recommendation on diagnosis in adults with left ventricular hypertrophy and genetic variants of unknown significance. Int J Cardiol 2014;177:400-408.

47. van der Tol L, Smid BE, Poorthuis BJ, et al. A systematic review on screening for Fabry disease: prevalence of individuals with genetic variants of unknown significance. J Med Genet 2014;51:1-9.

48. Apelland T, Gude E, Strøm EH, et al. Familial globotriaosylceramide-associated cardiomyopathy mimicking Fabry disease. Heart 2014;100:1793-1798.

49. Strømme P, Månsson JE, Scott H, Skullerud K, Hovig T. Encephaloneuropathy with lysosomal zebra bodies and GM2 ganglioside storage. Pediatr Neurol 1997:16:141-144.

50. Askari H, Kaneski CR, Semino-Mora C, et al. Cellular and tissue localization of globotriaosylceramide in Fabry disease. Virchows Arch 2007;451: 823-834.

51. Xu S, Lun Y, Brignol $N$, et al. Coformulation of a novel human $\alpha$-galactosidase a with the pharmacological chaperone AT1001 leads to improved substrate reduction in Fabry mice. Mol Ther 2015;23:1169-1181.

52. Roy S, Touboul D, Brunelle A, et al. [Imaging mass spectrometry: a new tool for the analysis of skin biopsy. Application in Fabry's disease]. Ann Pharm Fr 2006;64:328-334

53. Fuller M, Sharp PC, Rozaklis T, et al. Urinary lipid profiling for the identification of fabry hemizygotes and heterozygotes. Clin Chem 2005;51:688-694. 